\title{
THE VITALI-HAHN-SAKS THEOREM FOR BOOLEAN ALGEBRAS WITH THE SUBSEQUENTIAL INTERPOLATION PROPERTY
}

\author{
FRANCISCO JOSÉ FRENICHE ${ }^{1}$
}

\begin{abstract}
It is shown that the Vitali-Hahn-Saks theorem holds for a new class of Boolean algebras which are defined by a separation property of its disjoint sequences: the Subsequential Interpolation Property. It is also proved that this property is strictly weaker than the Interpolation Property, the $(f)$ Property and the Subsequential Completeness Property.
\end{abstract}

In $[2$, p. 36] the problem is raised of finding an algebraic characterization of those Boolean algebras for which the Vitali-Hahn-Saks theorem holds.

Some results in this direction are known: in [6 and 3] it is proved that this theorem is true for Boolean algebras with the Interpolation Property, in [5] the same result is proved for those which have the $(f)$-Property, and in [4] it is announced that the theorem is true for the class of Boolean algebras with the Subsequential Completeness Property.

In this paper we define a new class of Boolean algebras (Definition 1), strictly containing the above-mentioned classes (Proposition 2 and Theorem 7), and such that the Vitali-Hahn-Saks theorem holds for measures on these Boolean algebras (Theorem 4).

DEFINITION 1. Let $A$ be a Boolean algebra. We say that $\mathcal{A}$ has the Subsequential Interpolation Property if, for every disjoint sequence $\left(a_{n}\right)_{n \in \omega}$ in $A$ and for every infinite $M \subset \omega$, there exist $a \in A$ and an infinite $N \subset M$ such that $a_{n} \leq a$ if $n \in N$ and $a_{n} \wedge a=0$ if $n \in \omega-N$.

Proposition 2. If a Boolean algebra $A$ has one of the following properties:

(1) Interpolation Property,

(2) (f)-Property,

(3) Subsequential Completeness Property,

then $A$ also has the Subsequential Interpolation Property.

ProOF. We first suppose that $\mathcal{A}$ has the $(f)$-Property. Let $\left(a_{n}\right)_{n \in \omega}$ be a disjoint sequence in $A$ and $M$ be an infinite subset of $\omega$. We apply (2) to the sequences $\left(a_{n}\right)_{n \in M}$ and $\left(a_{n}\right)_{n \in \omega-M}$, taking an infinite $N_{1} \subset M$ and $b_{1} \in \mathcal{A}$ such that $a_{n} \leq b_{1}$ if $n \in N_{1}$ and $a_{n} \wedge b_{1}=0$ if $n \in \omega-M$, and for each $N \subset N_{1}, a_{N} \in \mathcal{A}$ such that $a_{n} \leq a_{N}$ if $n \in N$ and $a_{n} \wedge a_{N}=0$ if $n \in N_{1}-N$.

We consider the sequences $\left(a_{n}\right)_{n \in M-N_{1}}$ and $\left(a_{n}\right)_{n \in N_{1}}$. By (2) we can choose an infinite $N \subset N_{1}$ and $b_{2} \in \mathcal{A}$ such that $a_{n} \leq b_{2}$ if $n \in N$ and $a_{n} \wedge b_{2}=0$ if

Received by the editors June 27, 1983.

1980 Mathematics Subject Classification. Primary 28A33; Secondary 06E10, 28A60.

Key words and phrases. Boolean algebra, uniformly strongly additive sequence of measures.

${ }^{1}$ This is a slightly modified part of the author's Ph.D. Thesis prepared at the Universidad de Sevilla under the direction of Professor Juan Arias de Reyna. I wish to thank Professor Arias de Reyna for his interest and advice. 
$n \in M-N_{1}$. It is now easy to check that if $a=b_{1} \wedge b_{2} \wedge a_{N}$, then $a \wedge a_{n}=0$ if $n \in \omega-N$ and $a \geq a_{n}$ if $n \in N$. Thus (2) implies the Subsequential Interpolation Property. Since obviously (1) implies (2), we have also proved that (1) implies the same property.

Suppose now that $A$ has the Subsequential Completeness Property and $\left(a_{n}\right)_{n \in \omega}$ and $M$ are as above. We choose an infinite $N \subset M$ such that there exists in $A$ the least upper bound $a$ of $\left(a_{n}\right)_{n \in N}$. It is clear that $a$ verifies $a \wedge a_{n}=0$ if $n \in \omega-N$ and $a_{n} \leq a$ if $n \in N$, and this completes the proof.

LEMMA 3. Let $\mathcal{A}$ be a Boolean algebra with the Subsequential Interpolation Property. For every $\varepsilon>0$, every disjoint sequence $\left(a_{n}\right)_{n \in \omega}$ in $\mathcal{A}$, and every complexvalued bounded variation finitely additive measure $\mu$ on $A$, there exist $a \in A$ and an infinite $M \subset \omega$ such that $|\mu|(a)<\varepsilon, a_{n} \wedge a=0$ if $n \in \omega-M$ and $a_{n} \leq a$ if $n \in M$.

PROOF. Let $\left(M_{k}\right)_{k \in \omega}$ be a disjoint sequence of infinite subsets of $\omega$. By the Subsequential Interpolation Property we can choose for each $k \in \omega$ an infinite $N_{k} \subset M_{k}$ and $b_{k} \in A$ such that $a_{n} \leq b_{k}$ if $n \in N_{k}$ and $a_{n} \wedge b_{k}=0$ if $n \in \omega-N_{k}$.

Let $c_{k}=b_{k}-\bigvee\left\{b_{i}: i<k\right\}$ for $k \in \omega$. Since $\left(c_{k}\right)_{k \in \omega}$ is a disjoint sequence in $\mathcal{A}$, we have $\lim _{k \rightarrow \infty}|\mu|\left(c_{k}\right)=0$. Hence there exists $k \in \omega$ such that $|\mu|\left(c_{k}\right)<\varepsilon$.

It is clear that $a=c_{k}$ and $M=N_{k}$ satisfy the conclusion of the lemma.

THEOREM 4. Let $A$ be a Boolean algebra with the Subsequential Interpolation Property, and let $\left(\mu_{n}\right)_{n \in \omega}$ be a sequence of complex-valued bounded variation finitely additive measures on $A$ such that $\lim _{n \rightarrow \infty} \mu_{n}(a)=0$ for every $a \in \mathcal{A}$. Then $\left(\mu_{n}\right)_{n \in \omega}$ is uniformly strongly additive.

PROOF. Assume that $\left(\mu_{n}\right)_{n \in \omega}$ is not uniformly strongly additive. We can suppose, by passing to a subsequence if necessary, that there exist an $\varepsilon>0$ and a disjoint sequence $\left(a_{n}\right)_{n \in \omega}$ in $A$ such that $\left|\mu_{n}\left(a_{n}\right)\right| \geq \varepsilon$ for every $n \in \omega$.

Let $n_{0}=0$ and suppose, by recurrence, that $k \in \omega$ and we have defined $n_{j} \in \omega$, $N_{j} \subset \omega$, and $b_{j} \in \mathcal{A}$ for $j \leq k$ such that the following conditions are satisfied:

(a) $n_{j} \in N_{j-1}, n_{j}<\min \left(N_{j}\right)$;

(b) $N_{j-1} \supset N_{j}, b_{j-1} \geq b_{j}$ and $N_{j}$ is infinite;

(c) $b_{j} \wedge a_{n}=0$ if $n \in \omega-N_{j}$ and $a_{n} \leq b_{j}$ if $n \in N_{j}$;

(d) $\left|\mu_{n_{j}}\right|\left(b_{n_{j}}\right)<\varepsilon / 3$ and $\sum_{i<j}\left|\mu_{n_{j}}\left(a_{n_{i}}\right)\right|<\varepsilon / 3$

for every $j \leq k$, where $N_{-1}=\omega$ and $b_{-1}=1$ (the unit element of $\mathcal{A}$ ).

Since $\lim _{n \rightarrow \infty} \mu_{n}(a)=0$ for every $a \in A$ and $N_{k}$ is infinite, there exists $n_{k+1} \in$ $N_{k}$ such that $\sum_{i<k}\left|\mu_{n_{k+1}}\left(a_{n_{i}}\right)\right|<\varepsilon / 3$.

By Lemma 3 there exist $b_{k+1} \in A$ and an infinite $N_{k+1} \subset N_{k}$ such that $\left|\mu_{n_{k+1}}\right|\left(b_{k+1}\right)<\varepsilon / 3, a_{n} \leq b_{k+1}$ if $n \in N_{k+1}$ and $a_{n} \wedge b_{k+1}=0$ if $n \in N_{k}-N_{k+1}$. We can further assume that $n_{k+1}<\min \left(N_{k+1}\right)$ and $b_{k+1} \leq b_{k}$, and continue the induction.

Consider the following family of elements of $A$ : $a_{n_{0}}, a_{n_{1}}, \ldots, a_{n_{k}}, \ldots$, $-\left(a_{n_{0}} \vee b_{0}\right), b_{0}-\left(a_{n_{1}} \vee b_{1}\right), \ldots, b_{k-1}-\left(a_{n_{k}} \vee b_{k}\right), \ldots$ We claim this family is disjoint. Indeed, if $j>k$ then $n_{j} \in N_{j-1} \subset N_{k}$ and $a_{n_{j}} \leq b_{k}$, hence $a_{n_{j}} \wedge\left(b_{k-1}-\left(a_{n_{k}} \vee b_{k}\right)\right)=$ 0 , and since $j-1 \geq k$ then $b_{j-1} \leq b_{k}$, hence

$$
\left(b_{j-1}-\left(a_{n_{j}} \vee b_{j}\right)\right) \wedge\left(b_{k-1}-\left(a_{n_{k}} \vee b_{k}\right)\right)=0 .
$$

If $j<k$ then $n_{j} \in \omega-N_{j}$ and $N_{j} \supset N_{k-1}$, hence $b_{k-1} \wedge a_{n_{j}}=0$. 
Since $A$ has the Subsequential Interpolation Property, there exist an infinite $M \subset \omega$ and $a \in A$ such that $a_{n_{k}} \leq a$ if $k \in M, a_{n_{k}} \wedge a=0$ if $k \in \omega-M$, and $a \wedge\left(b_{k-1}-\left(a_{n_{k}} \vee b_{k}\right)\right)=0$ for all $k \in \omega$.

Fix $k \in M$. We have

$$
\begin{aligned}
\mu_{n_{k}}(a)= & \mu_{n_{k}}\left(a_{n_{k}}\right)+\mu_{n_{k}}\left(\bigvee\left\{a_{n_{i}}: i \in M, i<k\right\}\right) \\
& +\mu_{n_{k}}\left(a-\bigvee\left\{a_{n_{i}}: i \in M, i \leq k\right\}\right)
\end{aligned}
$$

By construction,

$$
a \leq-\left(b_{i-1}-\left(a_{n_{i}} \vee b_{i}\right)\right)=\left(-b_{i-1}\right) \vee a_{n_{i}} \vee b_{i}
$$

for every $i \leq k$, hence

$$
a \leq \bigwedge_{i \leq k}\left(\left(-b_{i-1}\right) \vee a_{n_{i}} \vee b_{i}\right)=a_{n_{0}} \vee \cdots \vee a_{n_{k}} \vee b_{k}
$$

Thus

$$
a-\bigvee\left\{a_{n_{i}}: i \in M, i \leq k\right\}=a-\bigvee\left\{a_{n_{i}}: i \leq k\right\} \leq b_{k}
$$

Finally,

$$
\begin{aligned}
\left|\mu_{n_{k}}(a)\right| \geq & \left|\mu_{n_{k}}\left(a_{n_{k}}\right)\right|-\left|\sum\left\{\mu_{n_{k}}\left(a_{n_{i}}\right): i \in M, i<k\right\}\right| \\
& -\left|\mu_{n_{k}}\left(a-\bigvee\left\{a_{n_{i}}: i \in M, i \leq k\right\}\right)\right| \\
\geq & \left|\mu_{n_{k}}\left(a_{n_{k}}\right)\right|-\sum_{i<k}\left|\mu_{n_{k}}\left(a_{n_{i}}\right)\right|-\left|\mu_{n_{k}}\right|\left(b_{k}\right) \geq \varepsilon-\frac{\varepsilon}{3}-\frac{\varepsilon}{3}=\frac{\varepsilon}{3} .
\end{aligned}
$$

Since $M$ is infinite, we cannot have $\lim _{n \rightarrow \infty} \mu_{n}(a)=0$; that is a contradiction.

We shall now prove that the class of Boolean algebras with the Subsequential Interpolation Property is larger than the other classes mentioned above. We use a method of R. Haydon [4] for constructing Boolean algebras with the Subsequential Completeness Property and other special properties.

LEMMA 5. Let $\left(D_{n}\right)_{n \in \omega}$ be a disjoint sequence of infinite subsets of $\omega$ which covers $\omega$. If $A_{0}$ is the Boolean algebra of subsets of $\omega$ generated by $\left(D_{n}\right)_{n \in \omega}$ and by the finite subsets, then there is no $A \in A_{0}$ such that $\left\{n \in \omega: A \cap D_{n}\right.$ is finite $\}$ and $\left\{n \in \omega: D_{n}-A\right.$ is finite $\}$ are both infinite.

ProOF. Notice first that if $B$ is the Boolean algebra generated by the sequence $\left(D_{n}\right)_{n \in \omega}$ then the elements of $B$ have the form $\bigcup\left\{D_{n}: n \in N\right\}$, where $N$ is a finite or cofinite subset of $\omega$.

It is clear that $\mathcal{A}_{0}=\{A \subset \omega: B \Delta A$ is finite for some $B \in B\}$. Thus if for some $A \in A_{0}$ the sets $\left\{n \in \omega: A \cap D_{n}\right.$ is finite $\}$ and $\left\{n \in \omega: D_{n}-A\right.$ is finite $\}$ are both infinite, then $\left\{n \in \omega: B \cap D_{n}\right.$ is finite $\}$ and $\left\{n \in \omega: D_{n}-B\right.$ is finite $\}$ are also infinite for the corresponding $B \in B$, contradicting the above expression for $B$.

Proposítion 6. Let $\left(D_{n}\right)_{n \in \omega}$ and $A_{0}$ be as in Lemma 5. There exists a Boolean algebra $A$ of subsets of $\omega$ with the Subsequential Completeness Property, 
containing $A_{0}$, such that for every infinite $N \subset \omega$ there is $M \subset N$ such that for no $A \in \mathcal{A}, A \cap D_{n}$ is finite if $n \in M$ and $D_{n}-A$ is finite if $n \in N-M$.

ProOF. With an argument similar to that of $[4,1 \mathrm{D}, 1 \mathrm{E}], \mathcal{A}$ can be constructed as the union of an increasing transfinite sequence $\left(A_{\alpha}\right)_{\alpha<2^{\omega}}$ of Boolean algebras of subsets of $\omega$, starting with $A_{0}$ and using the following

Claim. Let $B$ be a Boolean algebra of subsets of $\omega$ with $|B|<2^{\omega}$ and assume that there exists a family $\left(M_{i}, N_{i}\right)_{i \in I}$, with $M_{i} \subset N_{i} \subset \omega$ for all $i \in I$ and $|I|<2^{\omega}$, such that for no $B \in B$ and no $i \in I$ we have $B \cap D_{n}$ is finite if $n \in M_{i}$ and $D_{n}-B$ is finite if $n \in N_{i}-M_{i}$.

Then, given any disjoint sequence $\left(B_{n}\right)_{n \in \omega}$ in $B$, there exists an infinite $M \subset \omega$ such that for no $B \in B_{M}$ and no $i \in I$ we have $B \cap D_{n}$ is finite if $n \in M_{i}$ and $D_{n}-B$ is finite if $n \in N_{i}-M_{i}$, where $B_{M}$ is the Boolean algebra generated by $B$ and the set $\bigcup\left\{B_{n}: n \in M\right\}$.

THEOREM 7. There exists a Boolean algebra with the Subsequential Interpolation Property which has neither the Subsequential Completeness Property nor the (f)-Property.

Proof. Let $\left(D_{n}\right)_{n \in \omega}$ and $A$ be as in Proposition 6 , and define $B$ as the quotient of $\mathcal{A}$ under the ideal of finite subsets of $\omega$.

Firstly, $B$ has the Subsequential Interpolation Property. Indeed, let $\left(b_{i}\right)_{i \in \omega}$ be a disjoint sequence in $B$ and let $C_{i} \in b_{i}$ and $A_{i}=C_{i}-\bigcup\left\{C_{j}: j<i\right\}$ for $i \in \omega$.

By disjointness, $C_{i} \cap C_{j}$ is finite for $i \neq j$, hence $A_{i} \in b_{i}$. Since $A$ has the Subsequential Completeness Property and the sequence $\left(A_{i}\right)_{i \in \omega}$ is disjoint, given an infinite $M \subset \omega$, there is an infinite $N \subset M$ such that there exists in $A$ the least upper bound $A$ of $\left(A_{i}\right)_{i \in N}$. It is clear that if $b$ is the class of $A$, then $b_{i} \leq b$ if $i \in N$ and $b_{i} \wedge b=0$ if $i \in \omega-N$. (Notice that the class of Boolean algebras which have the Subsequential Interpolation Property is closed under quotients.)

$B$ does not have the $(f)$-Property. Otherwise, by considering the sequences $\left(b_{n}\right)_{n \in \omega}$ and $\left(d_{n}\right)_{n \in \omega}$, where $b_{n}$ is the class of the empty set and $d_{n}$ is the class of $D_{n}$, for $n \in \omega$, we can choose an infinite $N \subset \omega$ such that for every $M \subset N$ there exists $b_{M} \in B$ with $d_{n} \wedge b_{M}=0$ if $n \in M$ and $d_{n} \leq b_{M}$ if $n \in N-M$. Let $A_{M} \in b_{M}$ for $M \subset N$. We have $D_{n} \cap A_{M}$ is finite if $n \in M$ and $D_{n}-A_{M}$ is finite if $n \in N-M$. However, by construction, for every infinite $N \subset \omega$ there exists $M \subset N$ such that for no $A \in A$ we have $D_{n} \cap A$ is finite if $n \in M$ and $D_{n}-A$ is finite if $n \in N-M$.

Finally, $B$ does not have the Subsequential Completeness Property. Indeed, let $\left(d_{n}\right)_{n \in \omega}$ be as above. We prove that for no infinite $M \subset \omega,\left(d_{n}\right)_{n \in M}$ has least upper bound in $B$. To see this let $b$ be an upper bound of $\left(d_{n}\right)_{n \in M}, A \in b$, and for each $n \in M$, let $m_{n} \in D_{n} \cap A$. Since the sequence $\left(\left\{m_{n}\right\}\right)_{n \in M}$ is disjoint and $A$ has the Subsequential Completeness Property, there exists an infinite $N \subset M$ such that there exists in $A$ the least upper bound $C$ of $\left(\left\{m_{n}\right\}\right)_{n \in N}$. It is clear that $C=\left\{m_{n}: n \in N\right\}$ and that the class of $A-C$ is an upper bound of $\left(d_{n}\right)_{n \in M}$ that is strictly smaller than $b$.

REMARK. F. K. Dashiell [1] has shown that the Vitali-Hahn-Saks theorem holds for up-down semicomplete Boolean algebras which verify an additional lattice property. It is easy to find examples of Boolean algebras with the Subsequential 
Interpolation Property without the Dashiell property. However it is unknown to us if our property is weaker than that of Dashiell.

\section{REFERENCES}

1. F. K. Dashiell, Non weakly compact operators from order-Cauchy complete $C(S)$ lattices, with applications to Baire classes, Trans. Amer. Math. Soc. 266 (1981), 397-413.

2. J. Diestel and J. J. Uhl, Jr., Vector measures, Math. Surveys, No. 15 Amer. Math. Soc., Providence, R.I., 1977.

3. B. T. Faires, On Vitali-Hahn-Saks-Nikodým type theorems, Ann. Inst. Fourier (Grenoble) 26 (1976), 99-114.

4. R. Haydon, A non reflexive Grothendieck space that does not contain $l_{\infty}$, Israel J. Math. 40 (1981), 65-73.

5. A. Moltó, On the Vitali-Hahn-Saks theorem, Proc. Roy. Soc. Edinburgh Sect. A 90 (1981), 163-173.

6. G. L. Seever, Measures on F-spaces, Trans. Amer. Math. Soc. 133 (1968), 267-280.

Facultad de Matemáticas, Universidad de Sevilla, C/Tarfia SN. Sevilla-12, SPAIN 